\title{
The oromaxillofacial rehabilitation in orthodontic-surgical protocols
}

\author{
Th. Gouzland ${ }^{1,2}$, M. Fournier ${ }^{1}$ \\ 1 Kinesiologist, specializing in oromaxillofacial rehabilitation \\ 2 Educator
}

\begin{abstract}
SUMMARY
Oro-maxillo-facial rehabilitation is an ancient practice that has developed over recent years through research and integration with physiotherapists in multidisciplinary teams, as is the case with orthodonticsurgical procedures. At the same time, the progress made in orthognathic and orthodontic surgery over the last 20 years encourages more and more patients to undergo surgery. Preoperative treatment is based on early assessment and preparation for optimal surgical conditions to come up with a functional plan. A short stay in a hospital, focusing on rehabilitation, is recommended. During the postoperative phase, the key objectives are to ensure the muscles and arteries all function perfectly, acceptance of the new face, and the immediate correction of any orofacial dyspraxia that has occurred during myofunctional therapy. The various specialists in this multidisciplinary team must constantly be in communication. The importance of postoperative physiotherapy will be illustrated by a study consisting of 35 cases of maxillomandibular osteotomy with orthodontic preparation and monitoring. The purpose of this study is to show occurrence of suboccipital and cervical muscle tensions as well as masticatory muscles. Then we will be able to see the importance of these practices, the impact on recovery, the impact on posture and how best to treat.
\end{abstract}

KEYWORDS

Physiotherapy, orthognathic surgery, orthodontic, myofunctional therapy, muscles, posture

INTRODUCTION

The progress made in the techniques and the results obtained by coupling orthognathic surgery with orthodontics allows the specialists to fix any functional and esthetic problems in patients affected by facial dysmorphia or major occlusal disorders. The patients benefit from a less-invasive surgery, with shorter recovery times and a more functional and comfortable recovery. This fits into the current social view where one's image is given greater importance. Similarly, a lot of research has been conducted on well-being and physical balance, which includes occlusal balance and balancing muscular tension. As a result, more and more patients are opting to have surgery.
Address for correspondence:

Thierry Gouzland Polyclinique Bordeaux-Tondu 145 rue du Tondu 33082 Bordeaux Cedex

E-mail: thierry.gouzland@icloud.com
This is an Open Access article distributed under the terms of the Creative Commons Attribution License (http://creativecommons.org/licenses/by/4.0), which permits unrestricted use, distribution, and reproduction in any medium, provided the original work is properly cited.
Article received: 30-09-2015. Accepted for publication: 30-10-2015. 


\section{The role of rehabilitation}

While the face is growing, the correlation between form and function is well documented $22,25,28$. This facial growth is influenced by predetermined genetic factors but also by acquired epigenetic factors. A correlation exists between the face and its orofacial functions. The structure allows the function to proceed and the function will then be modeled on the structure. A good functional dynamic facilitates a harmonious morphology. Similarly, dysfunctions contribute to the development of dysmorphic disorders. The orthognathic surgical teams coordinate with precise treatment plans to create new facial architecture and to restore an ideal dental occlusion. The desired occlusal balance and dental alignment are linked to muscular balance and functional orofacial symmetry $^{13}$. However, if orofacial dyspraxia is not corrected, there is an increased risk of delaying recovery or even causing a relapse ${ }^{10,12}$. The kinesiologist, the specialist in oromaxillofacial rehabilitation, should be placed at the center of the multidisciplinary team. Knowledge of the techniques and skills of each specialist as well as communication within the group are vital to obtaining the best results and to continue optimizing the care of the patients.

\section{Kinesiology}

The official definition, dating from 1946, describes the profession as paramedical, oriented toward massage. This became completely obsolete over the years, especially because it was out of touch with the developments and techniques in the area and with medicine in general. In 2015, a new definition was adopted by the National Assembly. Such terms as: prevention, health promotion, diagnosis, and university research emerged, but of particular importance was the "treatment of movement disorders and deficiencies or alterations in functional capacity," which corresponds to the treatment of the orofacial sphere. The professional, following a diagnostic assessment, has the freedom to choose what to do and takes responsibility for his actions.

\section{Orofacial myofunctional rehabilitation}

As with any orthopedic surgery, rehabilitative care provides a comfortable postoperative recovery in terms of managing the pain, schedule, and quality of life of the patients. However, the complexity of the cephalic region requires specific training. The face is a vitally important functional site. Significant functions of the face include respiration, chewing, swallowing, speaking, and displaying emotions, and the face is linked to overall posture. Any dysfunction will negatively affect this functional equilibrium. Even if it falls within our field of expertise and professional nomenclature, the acquisition of specific anatomical, physiopathological, and technical skills is still necessary. These will add to our physical therapy abilities as well as our general approach to the patient and the specific vision of the kinesiologist.

Although interest in this specialty is developing, there are only a few trained kinesiologists. Orofacial myofunctional 
rehabilitation, referred to as myofunctional therapy in the English-speaking world, is often referred to as lingual rehabilitation in France. This term appears to be quite narrow as our focus is not only on lingual dyspraxia but also on the platysma facial muscles, the masticatory muscles, the temporomandibular joints (TMJs) and so on. It is, therefore, the vision of this maxillofacial specialty which originated in France in the 1960s with Mr Fournier ${ }^{10}$. After obtaining his degree, Mr Fournier met Professor Delaire, a maxillofacial surgeon. His problem was managing the tongue in the event of orthognathic surgical relapse. Over time and with the support of Professor Delaire, these concepts and approaches to therapy were developed. Meanwhile, in the '70s and '80s, in the United States, Straub and Garliner developed their work on facial exercises with faults in pronunciation as their main focus.

\section{Physiology}

Physiological knowledge improves our understanding of the mechanisms associated with rehabilitation. When it comes to the muscular components, the functional equilibrium between the agonistic and antagonistic muscles must be re-established. In cases of muscular hypotonia, a muscular reinforcement is carried out, which is well outlined by Guimaraes ${ }^{14}$. Reinforcement without movement is isometric and with movement is isotonic. An isotonic movement is deemed concentric if the muscle contracts or eccentric if the muscle extends. It is, therefore, a hindrance to movement. This makes it easier to choose the type of exercise according to the function or action of the muscle being treated. When it comes to contracted, spastic, or hypertonic muscles, muscle relaxation is necessary. This can be achieved with physical therapy using the techniques described by Alvarado². We can also draw upon Schultz's relaxation techniques (known as autogenic training), cited by Girard and Leroux ${ }^{13}$ and also by Alvarado ${ }^{1}$. This type of technique is also efficient in fixing certain parafunctions, such as bruxism. Regarding the functional aspects, erroneous functions must be reprogrammed and above all must be automatized. This capacity for the acquisition or modification of new motor patterns is explained by the brain's neuronal plasticity. Moeller and Paskay ${ }^{25}$ have prepared some guidelines concerning this process. First of all, the function must be used regularly or else the cortical representation of somatosensation of these muscles is decreased. The movement must be as specific as possible and must be repeated so that it becomes natural. It must also be increased in intensity to maintain its long-term well-being. They also discuss transference-i.e., when a function is automatized, other associated functions improve. Interference, on the other hand, is a return to the erroneous movement that continues to be triggered despite the functional correction. Therefore the kinesiologist can use all these guidelines to find a functional balance during the orthodontic treatment and the recovery. 


\section{PREOPERATIVE PHASE}

Once the orthognathic surgical decision is decided upon by both the orthodontist and the surgeon, the treatment plan is put in place. The orthodontist now tries to align the dental arches in anticipation of a osteotomy. The objective of rehabilitation will be to participate in the patient's musculoarticular function (Fig. 1). Treatment is organized following the initial assessment ${ }^{4}$. The surgeon requests an assessment of the orofacial dyspraxia to evaluate the severity of the dysfunction and the postoperative risks. However, the orthodontist can request the assistance of the kinesiologist a lot earlier in the process if he judges that a patient has a dysfunction that may interfere with his treatment. This phase lasts an average of 18 months, rehabilitation can begin at any point during this period. We note that an early assessment improves how best to organize the time management. This is to avoid having, for example, urgent surgical preparation just 1 month before surgery. Inversely, in the case of a favorable assessment, rehabilitation may be delayed to immediately before hospitalization.

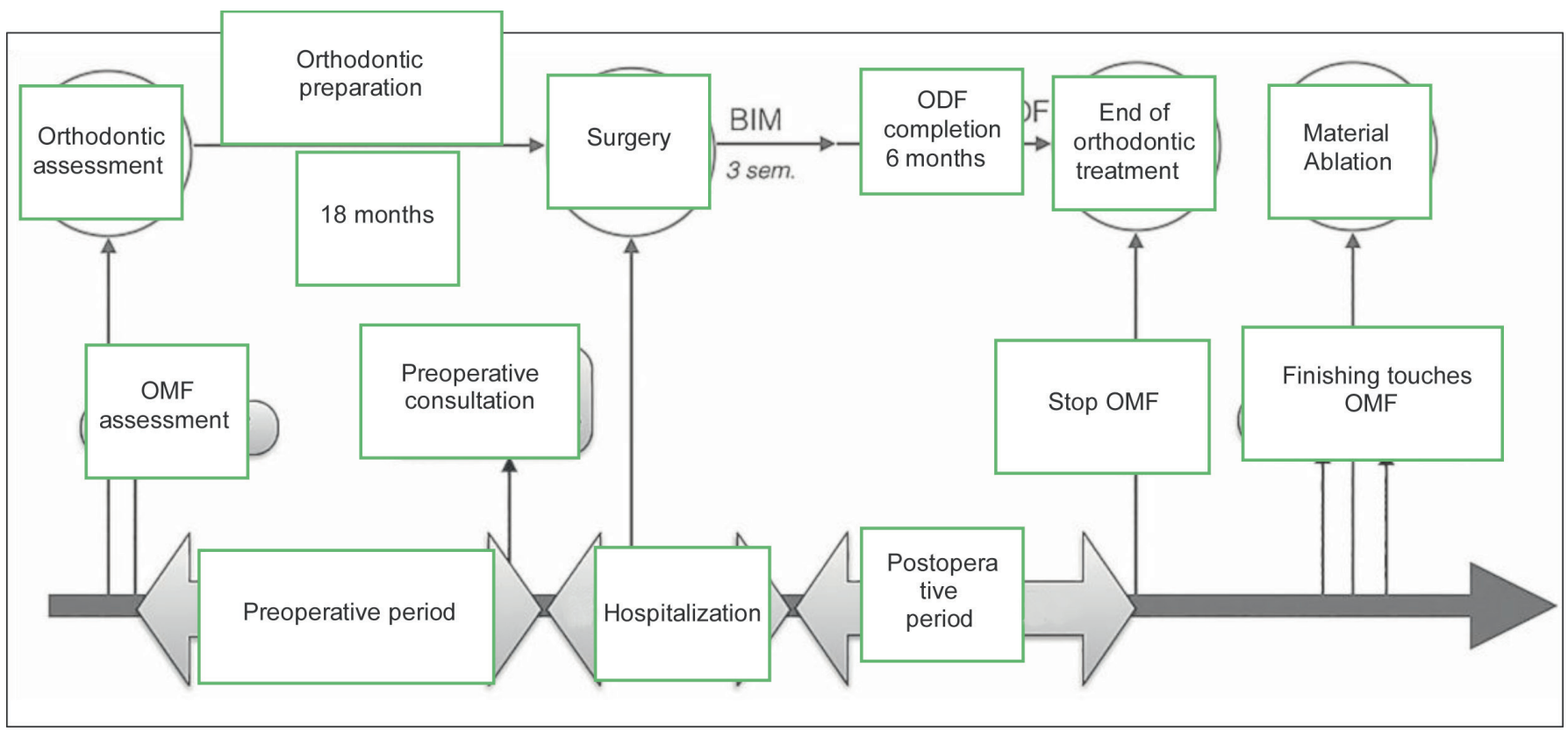

Figure 1

Coordinated surgical physical and orthodontic sequence of the patient. 


\section{Assessment and diagnosis}

An evaluation of the different orofacial functions, as well as an evaluation of the musculoskeletal system, can show whether preoperative rehabilitation is needed, facilitate the organization of time, and individualize our therapeutic strategy for each patient. Being aware of the dysfunctions shown by these tests is the basis for all corrections.

\section{Resting posture of the tongue}

With all dyspraxias, the key aspect is the resting position of the tongue as described by Fournier ${ }^{10}$. The difference in pressure recorded by Engelke ${ }^{8}$ in the subpalatal zone permits a true effortless at-rest position where the apex of the tongue is on the retroincisive palatal papillae. Depending on the type of dysmorphia, characteristic positions have been found ${ }^{11}$ : in class II division 2 , the apex is placed on the floor of the mouth or on the lower incisors with the two posterior tiers placed on the palates. In class III, the tongue is low, spread out on the floor of the mouth. In class III, it is low, spread out on the base of the cheek (Fig. 2). In open bites, the tongue is interposed between the dental arches. Coded by Marchesan ${ }^{23}$, the lingual frenum test highlights its possible shortness. Very often one encounters tongues presenting absolute macroglossia, with a distorted contentcontainer relationship. Though rare, cases of lingual immaturity can be easily identified in patients who possess

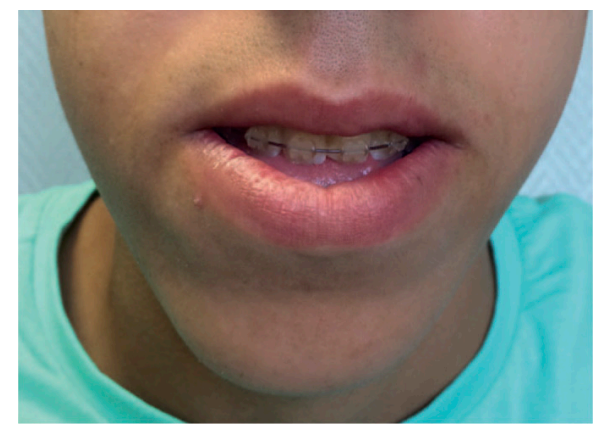

Figure 2

Class III lingual position. Tongue placed on the buccal floor, tip against the mandibular incisors.

neither intraoral proprioception nor awareness of tongue mobility.

\section{Ventilation or Breathing}

Often mistakenly referred to as respiration, ventilation is gaseous flow movement. It should be naso-nasal during the day and at night, while resting and while physically exerting oneself. A mixed or orally exclusive ventilation is frequently encountered and is attributed to dysmorphia as well as to bad habits. (Fig. 3a).

It is nonphysiological, acquired, and pathological. F. Susanibar ${ }^{31}$ describes a complete series of evaluations of the mechanics associated with ventilation. In addition, Rosenthal's functional test $^{29}$ and Guedin's nasal dyssynergy test exist. We are in agreement with Susanibar ${ }^{31}$ who advocates a modified Mallampati test (Fig. 4). The tongue must not be pulled when inspecting the available space in the oral cavity. All other findings such as snoring, nightly 
drooling, or dryness of the mouth are evaluated.

\section{Labial Function}

Analysis of the spontaneous labial position and the assessment of the orbicularis oris muscle in the lips provides evidence of possible labial incompetence. A hypotonic lower lip can be accompanied by a retracted upper lip, a marked chin furrow and even an involuntary contraction of the mentalis in labio-mental synkinesis. (Fig. 3b).

\section{Deglutition}

The function of deglutition is closely linked to the resting position of the tongue. Peristaltic movement in the anteroposterior direction must be done without contraction of the parasitic musculature, of the platysma facial muscles or contraction of the neck muscles. When a dysfunction related to deglutition is present, there may be labial aspiration, cheek salivation, and tongue propulsion, on the teeth or even inter-arch. There is often a dyssynergy associated with the masticator muscles which no longer stabilize the mandible in the position of maximal intercuspation. The pressure now exerted on the teeth close to 1500 times per day is significant and can result in a relapse.

\section{Phonation}

Phonation is also associated with the resting position of the tongue. Normally, there is no contact between the tongue and teeth. On the palatals, we should see lingual ascension close to retroincisive palatal papillae and not on the teeth. If any erroneous whistling or hissing occurs, inter-arch pulsion

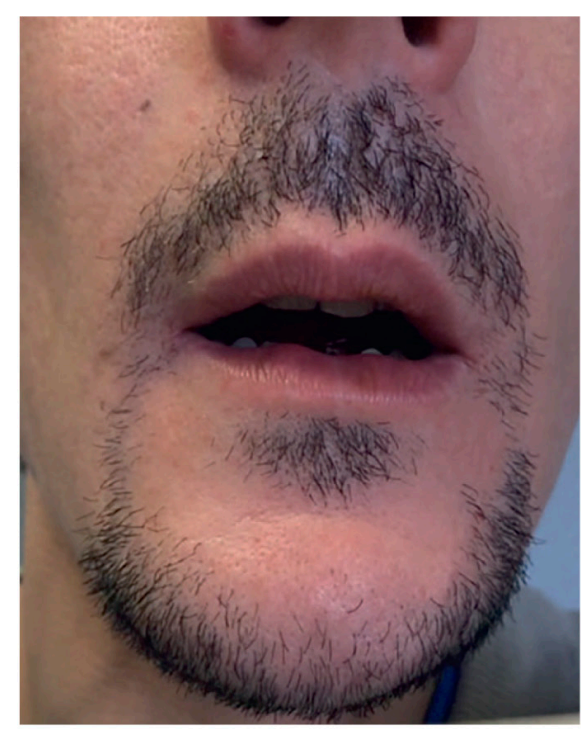

(a)

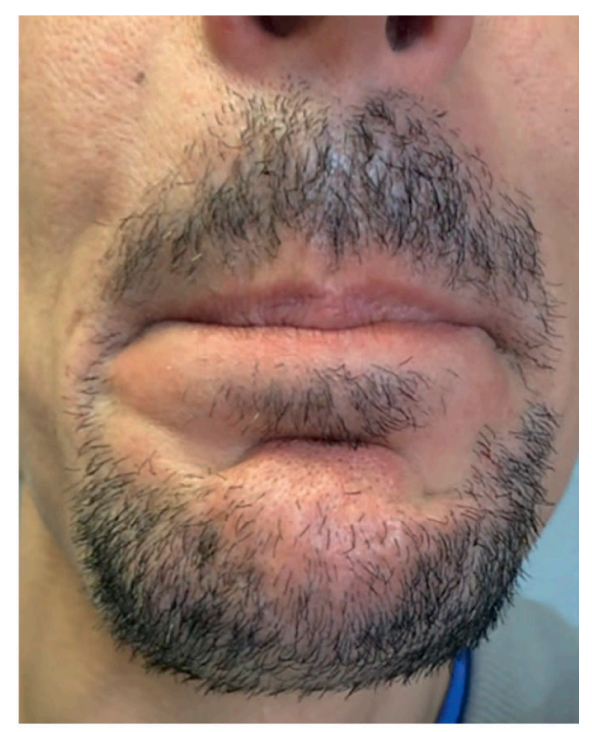

(b)

Figure 3

(a) Buccal or mixed ventilation. (b) Labiomental synkinesis: mental reflex contraction during voluntary lip-closing. 


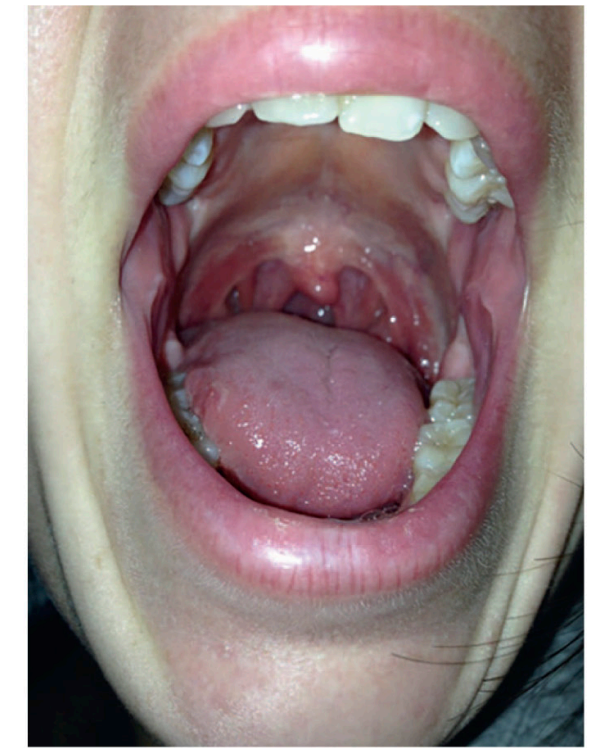

(a)

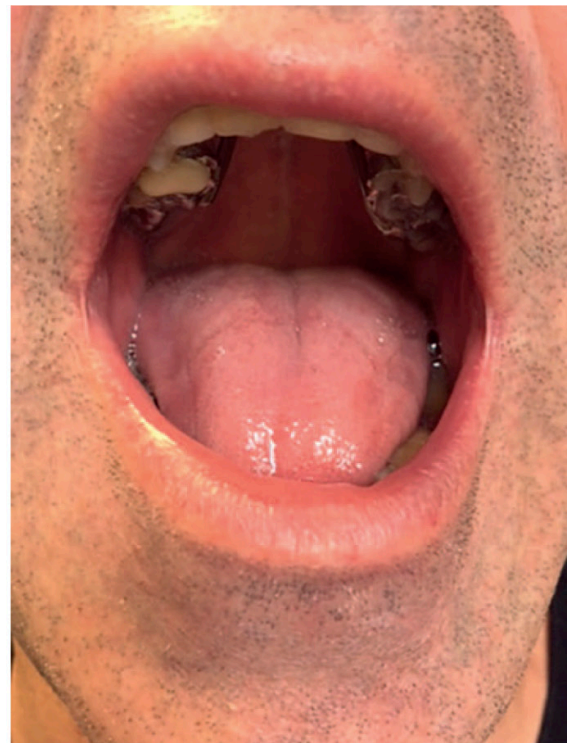

(b)

Figure 4

Modified Mallampati Scoring. (a) Score 1 with dental impression on the lateral edges of the tongue. (b) Score 4 with ogival palatal arch.

should be checked. The pressure exerted on the teeth is a bit constraining but it is important here to take note of the relationship between the different functions not only in terms of the assessment but also the treatment.

\section{Parafunctions}

They can be the root cause of excessive muscle tension, even temporomandibular dysfunction (TMD), but can equally be the cause of a range of orofacial dyspraxias. We may notice a pattern of nail-biting, lip-biting or cheek-biting, finger-sucking, tongue sucking, and excessive chewing of chewing-gum. Centered bruxism (jaw clenching) or off-centered (teeth grinding; Fig. 5) can lead to postoperative complications. These bad habits are numerous and are often found in stressful social and professional situations. Be aware that correcting these motor patterns and practicing relaxation techniques will decrease their onset and intensity.

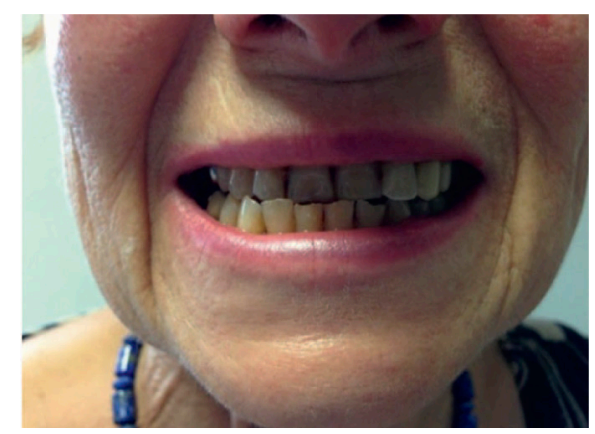

Figure 5 Aftereffects of Bruxism. 


\section{The Musculoskeletal System}

The kinematics of the TMJs is analyzed quantitatively and qualitatively. The amplitude of different movements is measured. We should investigate the presence of lateral deviations and articular sounds or pains. Palpation of the masticatory muscles and mandible depressor muscles indicates possible muscle contractures. The existence of a previous TMD can make following these guidelines more difficult. Treating this dysfunction improves comfort during orthodontia. Relaxing the muscle tension facilitates the work of the surgeon and makes the postoperative effects more tolerable.

\section{Posture}

A postural analysis should be performed. We will look at the different anatomical, neurological, and functional linkages between the masticatory apparatus and the patient's posture, mostly cervical but also general. This analysis is done morphostatically and if possible on a posture and gait analysis platform.

\section{Rehabilitation}

In terms of the assessment, if the patient seems balanced, a simple delayed preoperative consultation could suffice, even if the morphological changes will still require postoperative effort. Depending on the points highlighted during the evaluation, the treatment plan is put in place in conjunction with the findings of the other members of the team. Some patients have previously had rehabilitative kinesiology or speech therapy in adolescence. These patients already know how everything should function but have not automatized them. It will be a bit more difficult to analyze these functions and make them work.

It is a question of becoming aware of the area and the different structures involved in addition to the dysfunctions. The patient who understands will participate more actively and will automatically integrate these elements more readily. In a class III scenario, lingual rebound work must be done. In the class II case, the emphasis is on apical exercises and relaxing the back of the tongue (Fig. 6).

In the case of open bites, the motor pattern of the tongue must be worked so that it stops interposing itself, thereby facilitating the job of the orthodontist (Fig. 7). Lingual automatization is not possible when there is significant dysmorphia ${ }^{37}$ because the architectural changes modify the intraoral proprioception. This is confirmed during the postoperative phase. Nasal ventilation recovery provides postoperative comfort because of intermaxillary blocking and prevents any relapse in the long term. If the main reason for surgery is the presence of obstructive sleep apneahypopnea syndrome (OSAHS), this work takes precedence. If necessary, abdomino-diaphragmatic breathing must be corrected and nasal hygiene must be promoted. 


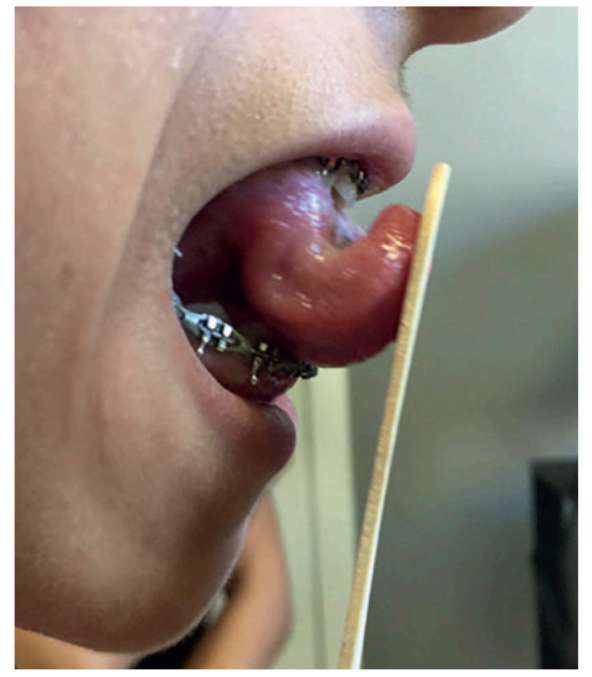

(a)

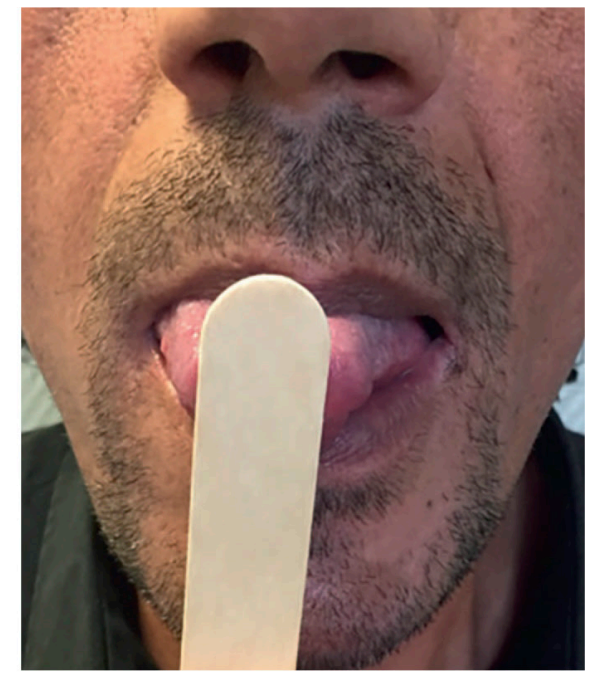

(b)

Figure 6

Example of a progressive reinforcement exercise of the lingual apex. (a) Incorrect position, tip hypotonic, back and base of the tongue hypertonic: the tongue is squashed. (b) Tongue in the correct position, the tip is toned, patient makes use of orbicularis lip muscle.

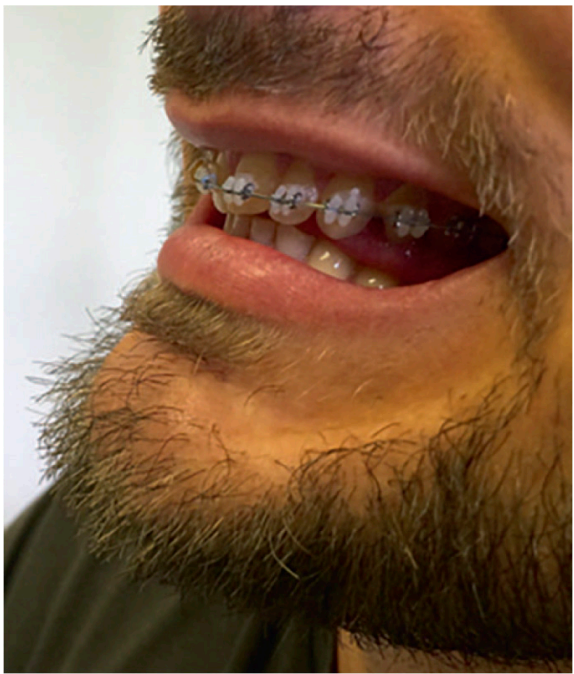

Figure 7

Tongue creeps into the open bite not only in phonation and deglutition but also at rest.

In the case of OSAHS, work is also done on the retrobasilingual zone and velar reinforcement can be performed.
(Fig. 8). The aim is for preoperative muscle equilibrium and normalization of the mandibular kinematics as far as possible. Technically, deep intraoral and extraoral massages are required as well as the removal of tension on the trigger points. Relaxation by stretching or even contractrelax relief of muscle tension are

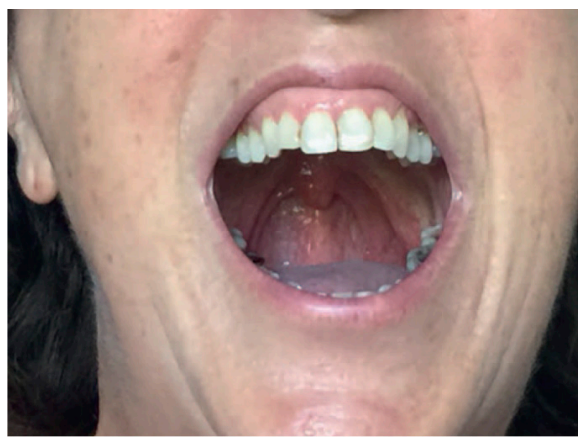

Figure 8

Example of velar and retrobasilingual reinforcement in the case of OSAHS. 
necessary. The hypotonia of the antagonistic muscles can be reinforced. However, if there is room, management of the parafunctions must be included.

\section{Preoperative consultation}

If a single consultation must be held because of logistics, organization, or other reasons, it must be this one. It ensures psychological preparation by informing the patient regarding what to expect in terms of postoperative effects: edemas, intermaxillary block-

\section{THE HOSPITALIZATION PHASE}

After months of preparation, the moment of surgical intervention arrives. It is generally anticipated although some patients can experience a certain level of anxiety. The surgery will radically modify the morphology of the face and mouth. This marks the end of the preparatory work carried out by the orthodontist and the kinesiologist. In this stage, we use new techniques with new objectives. This period, which lasts 3 days on average, emphasizes prodigal care and provides useful information. The rehabilitation begins on a patient who is bedridden, edematous (to some extent), and has partially transfixed muscles. The patient has intermaxillary blocking caused by rubber bands on the braces and brackets specially prepared by the orthodontist for surgery. In rare cases, this can be accomplished by miniscrew anchorages. The dental arches are also blocked with a previously-made ing, eating, nasal hygiene etc. We will then teach and make the patient aware of the resting position of the tongue. A breathing task is then performed as well as the alleviation or removal of muscle tension.

splint. The goal of rehabilitation during this phase is to make the postoperative stage as comfortable as possible for the patient and to recover a sound but early functionality to alleviate and shorten the length of treatment. The end of this phase is based on the condition that the patient will continue to manage their treatment.

\section{Respiration}

The first axis of intervention in kinesiology is respiratory. Immediate postoperative bronchial obstruction can require early intervention in the postanesthesia care unit (PACU) or hospital room. Fortunately such cases are rare. The surgeon or anesthesiologist then determines if the patients have a high risk of congestion (smokers...). In most cases, the signs of respiratory disturbance are rather linked to the sensation of having a lack of air brought on by elastic blocking and nasal congestion when the maxillary osteotomy has occurred. This phenomenon is better in patients who breathe buccally. Nasal mucositis and blood flow back into the naso-pharynx. Some patients because of transfixion or inhibition limit their swallowing of saliva. All together this will create a viscous mass deep in the throat, which will burden the patient. Respiratory kinesiotherapy now consists of achieving directed ventilation 
maneuvers, if necessary, that are associated with bronchial drainage. In these cases, we can resort to a bronchial aspiration or simply a buccal pumping. Learning to wash the nose with saline solution progressively cleans the upper airways; nose blowing is forbidden to avoid the risk of bleeding and of subcutaneous emphysema.

It is therefore vital to reinforce the instructions for resting position of the tongue in the new buccal cavity. The proprioceptive benchmarks will change, the first reflex is often to place the anterior part of the tongue toward the back of the teeth and to raise the retrobasilingual portion which will decrease respiratory flow. Relaxing the base of the tongue frees the aerodigestive crossroads and gives a better sensation so that the patient can swallow and communicate. Remounting the apex of the tongue as soon as the intraoral sensations are reawakened will permit the exploration of the new morphology and the immediate integration of functional proprioception and reflexes. If the dyspraxia is still present in the new layout, follow-up treatment will be longer and more complex. If the reference points are lost, it is better to start with a clean slate. If the buccal opening is blocked, this important exercise can be simply performed following verbal directions and without visual control.

\section{Edema}

The second objective of the treatment is to fight against edema and pain. Facial edema as well as the formation of a hematoma are a natural reaction to surgery (Fig. 9). Edemas vary from one individual to another in terms of volume and the duration of reabsorption. Patients find themselves very bloated quasi-systematically even if this is not the case. It is also important to follow the instructions on regular elevation and mobilization to enable drainage. Frequent and regular cryotherapy treatment promotes reabsorption and antalgia. It is recommended that this therapy be done regularly but it should not exceed 20 minutes so as to avoid expansion of the subjacent vascularization. ${ }^{27}$ The manoeuvers of manual lymphatic drainage (MLD) ${ }^{21}$, associated with early enlisting of the platysma facial muscles facilitate reabsorption ${ }^{7,37}$. Therefore, the patient is asked to communicate verbally as early as possible. Contracting the lips, tongue and cheek can be very efficient to drain tissue. Furthermore, talking is psychologically stimulating, which ensures that the patient does not feel isolated and experiences a certain return to "normalcy."

\section{Intermaxillary Blocking}

The third point concerns intermaxillary blocking. After initial radiography, with the surgeon's consent, we can perform the intermaxillary blocking procedure (Fig. 10). It is a very important step in the treatment, and it is very important also or the patient as well. It actually confirms the success of the procedure so far. All restraints are removed and the patient can look at themselves in the mirror, and the patient can see when the first movements have been made. At this point, the first progressive exercises are incorporated into the recovery In the autorehabilitation phase that will take place at home, the following are included: opening, propulsion, 


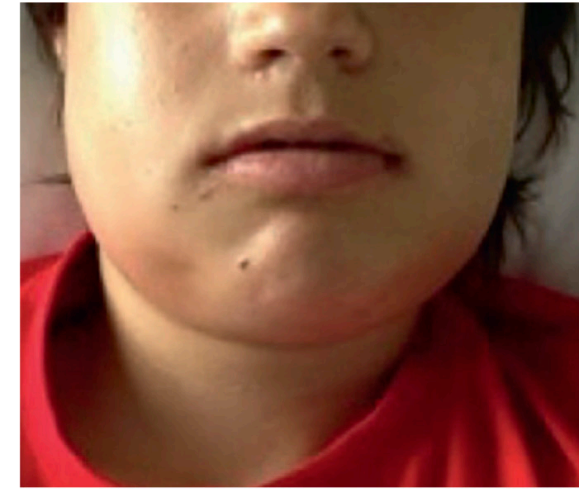

(a)

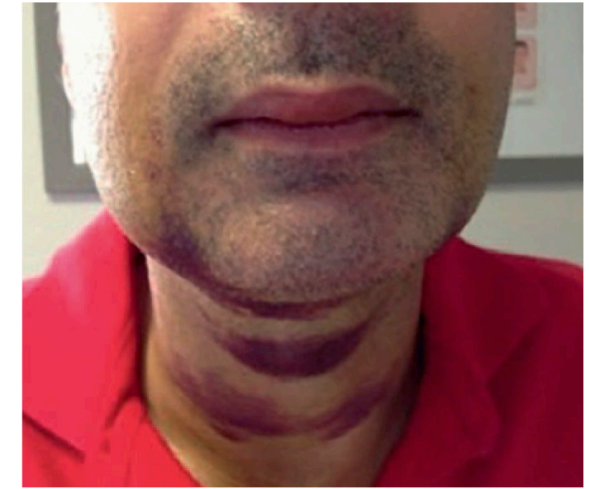

(b)

Figure 9

Edemas (a) and Hematomas (b) are significant postoperative side effects.

and separation, as well as removing the labial transfixion sutures. Many authors have expressed an interest in the early integration of a gentle and progressive rehabilitation. ${ }^{17,18,26,33,34}$ A new immobilizing technique is employed with two or three rubber bands which still allows the patient to move. No orthodontic correction needs to be induced to immobilize the patient. The objective is to integrate the new occlusion, to counteract "the muscle memory," and muscle tension during consolidation. The greater the risk of pulsion or lingual interposition, the more the blocking of the anterior incisor-canine will be encouraged.

\section{Therapeutic education}

Finally, the last role of the kinesiologist is to teach the patient. The patient can only leave the establishment if they are self-sufficient in his

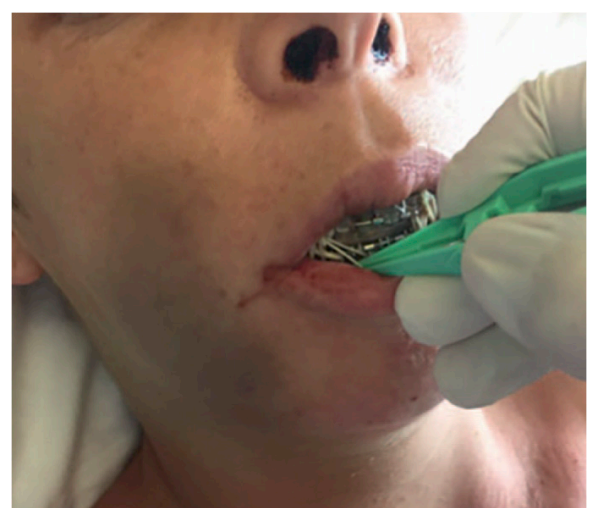

Figure 10

Ablation of the postoperative intermaxillary blocking.

nasal and buccal hygiene, in their management of the blockage, in their exercises, and in their alimentation. We will teach them the protocol for progressive unblocking, how much and how long for, a protocol for reintegration of chewing via food; liquids then mixed and finally solids. Not forgetting of course, the autorehabilitation protocol. 


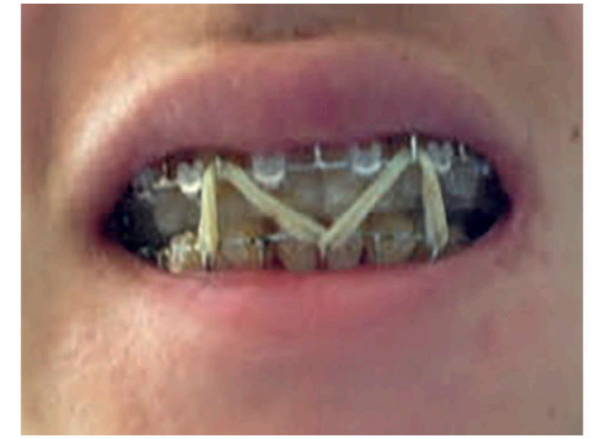

(a)

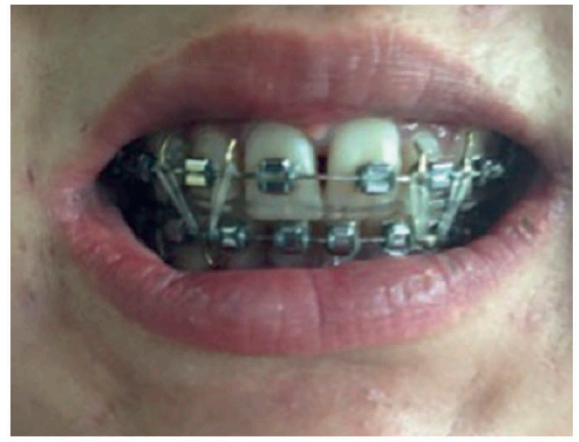

(b)

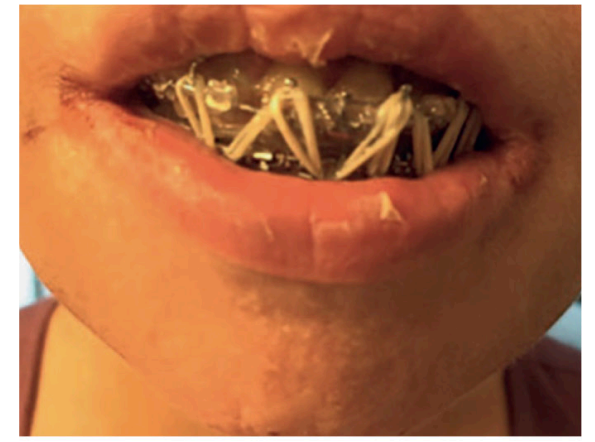

(c)

Figure $11 a, b, c$

Examples of intermaxillary blocking, 2 or 3 anterior rubber bands self-managed by the patient.

\section{POSTOPERATIVE PHASE}

Once the ideal facial architecture has been restored by surgery, this period will last 6 months, but this depends on the finishing touches to be performed by the orthodontist. Rehabilitation aims to:

- Monitor the postoperative followup;

- Contribute to antalgia;

- Decrease the postoperative edema;

- Relax the muscle tension;

- Restore mobility;

- Reinforce and automatize preoperative myofunctional activities;

- Integrate morphological changes.

\section{The first postoperative consultation}

The first consultation generally takes place during the first week after the patient's return home. The main goal is controlling any postoperative side effects and being able to interact quickly with other professionals in case of problems. On this occasion, the quality of the occlusion on the splint is checked while following up on the progressive unblocking program, with the focus on eating. In terms of physical work; the lymphatic drainage of the face, the superficial alleviation of intraoral and extraoral muscle tension, as well as cervicoscapular massages are performed. These two last items are detailed in a study presented later on. After confirming the completed autorehabilitation tasks, other exercises are prescribed to improve mobility, tongue position, and muscle relaxation. The prescribed exercises should be carried out three times per day. Any sign of excessive pain, infection, or discrepancy in relation to the occlusion could result in earlier than expected contact with the surgeon. The orthodontist should be contacted if problems should arise concerning, for example arcs, brackets, and hooks. As a general rule, the orthodontic finishing touches will be applied within 15 days to 3 weeks 


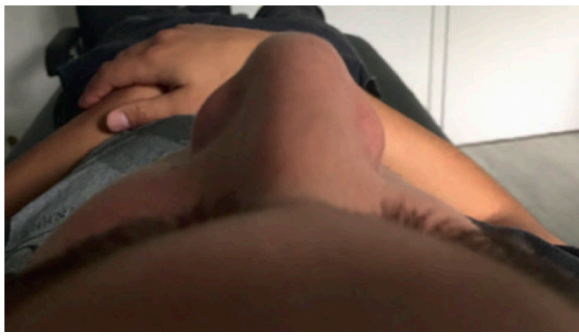

Figure 12

Asymmetric reabsorption of edema.

following surgery. During this period, the patient, as well as their family, may still have a number of questions and concerns, which must be addressed. These include issues related to the edema (if it is still severe), which is often asymmetrical in its reabsorption (Fig. 12), fatigue linked to surgical invasion as well as the patient's dietary needs/composition. The frequency of the visits now depends on the monitored clinical chart. It can be weekly, fortnightly, or every 3 weeks.

\section{Articular Mobility}

During treatment, the quality of mandibular mobility is reinforced to obtain (3 months later) a complete buccal opening of at least $40 \mathrm{~mm}$, permitting the patient to achieve normal daily functioning as well as the possibility of receiving future dental care. The earlier mobility is achieved, the better it will be. It is recommended that the opening should be a slightly greater than necessary given that after treatment stops, decreased stimulation will result in a closure of approximately 2-3 $\mathrm{mm}$. The proper

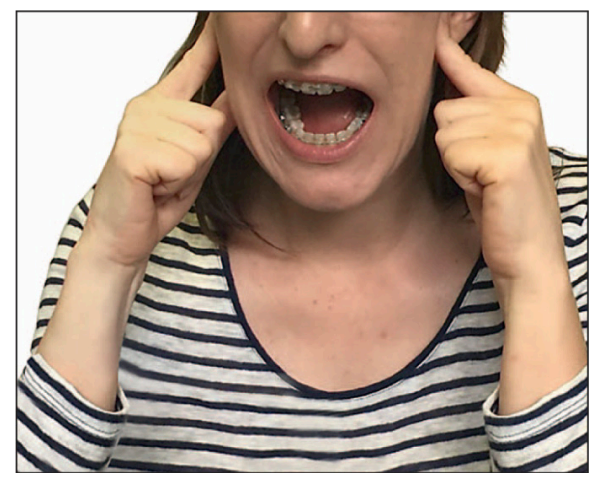

Figure 13

Example of the correction of mandibular kinematics with sensitive visual feedback.

kinematics of movement are recovered by observing themselves in a mirror, the patient's index fingers pointing toward their temporomandibular articulators (Fig. 13) to find out as much information as possible about the new mobility in the face.

\section{Facial Platysma muscles}

The exercising of the platysma facial muscle is essential. This guarantees the maximum amount of reabsorption of hematoma and edema. Their early stimulation allows the patient to show expressions on their new face. It also contributes to removing sensitive transfixions. In cases of significant buccal ventilation, the quick revival of the orbicularis oris and the relaxation of the mentalis muscle (associated with the automatization of naso-nasal ventilation) are crucial. Cases of labiomental synkinesis are frequent, this labial competence is crucial for achieving proper nasal ventilation.

It must be noted though that some patients are nasal respirators with a labial open bite. The long-term 
stability of the new occlusal structure is linked to a functional labiolingual equilibrium ${ }^{15}$.

\section{Mastication}

During treatment, the kinesiologist, specializing in oromaxillofacial rehabilitation, ensures the equilibrium of the orofacial functions. The early integration of intraoral proprioception after architectural changes is important. First of all, it integrates an adequate lingual resting position in the new buccal space. Whether the tongue is at rest or in use, it must not exert pressure on the dental arches nor must it be interposed between them. During musculoskeletal recovery, deglutition, ventilation, and phonation are then reevaluated and automatized. One of the major functions that has not been sufficiently explored in the literature in the specific case is mastication. It is, however, of primary importance because it stimulates contacts on sites that were not in use before the operation. Faulty recovery of mastication during the reintegration of eating patterns while the patient is convalescing can lead to preferential unilateral mastication. This leads to an increased risk of homolateral muscle contractures, and of slowing down the correct positioning and the contralateral meshing of teeth.

Makaremi and de Brondeau ${ }^{20}$ have observed the role of mastication in the correction of the occlusal quality. It is therefore important to be cautious about the kinds of foods ingested and occlusion, while ensuring that both sides are equally stimulated. Mastication is rarely bilateral, it is more often alternatingly unilateral. During early integration procedures, the important masticatory constraints are currently under review in the hopes of encouraging a faster, more efficient occlusal correction. The studies conducted by Takahashi $^{32}$ and de Valdés ${ }^{36}$ emphasize the link between the efficiency of the masticatory muscles and a high lingual position.

\section{Physical therapy}

Physical therapy is useful to liberate the tension of the masticatory musculature (Fig. 14). One can often identify the masseter muscles and medial and lateral pterygoid muscles this way. The temporal muscles are important when regaining masticatory function. Their tension often have a unilateral preponderance. According to Jouvin ${ }^{16}$, if this tension persist for too long, they will result in muscular fibrosis which will seriously limit the buccal opening. Manual work also

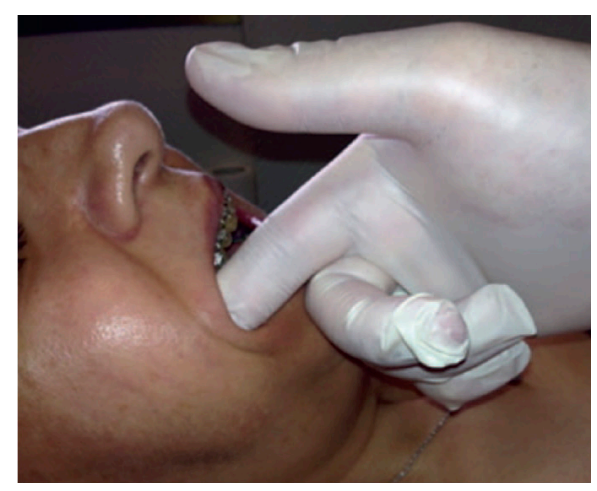

Figure 14

Removing intraoral muscle tension. 
allows the practitioner to act on the suboccipital and cervical muscle tension. Morphological modifications result in changes in tension on the suprahyoid and infrahyoid chains. The fibrosis may be submitted to excess tension that will have to be normalized to regain a balanced hyoglossal function; the hyoidal muscle system has an anatomical link with cervical posture. Its position also affects the ventilatory space. It is important to address it so as to maintain freedom of movement. The works of LeBanc show a return of the hyoid bone to its original position after some years. It is, therefore, important to try to stabilize it especially if the reason for surgery was the incidence of OSAHS.

\section{Posture}

The link between posture and maxillofacial structures, although rarely documented, is frequently outlined 1,6,24,28,30,37,38. We must, therefore, be aware of bad posture during convalescence and the integration of a new craniofacial architecture within the general framework of the body. A prolonged nocturnal unilateral position can cause constraints that will have very serious repercussions for TMJs. Cephalic anteriorization, interscapular amyotrophy, or cervical muscle tension can affect the axes of mandibular function, risking TMD. Recent analyses conducted by Faulin et al. ${ }^{9}$ conclude that posture does not influence the appearance of TMD, however, it is not a static analysis, just a position in a specific situation and does not factor in the notion of movement and of postural dynamics.

\section{Specific Cases}

Among the highlighted cases which must focus on and require specific care, we find traditional patients who were preoperative in class II and who were in the habit of compensating esthetically for years by lightly pushing the mandible. There are also those in class III who have lingual function on the buccal floor with the apex functioning in front of the maxillary dental arch.

The assumptions, frequent in postoperative though transitory, are more or less present from one patient to the other and last a reasonably longtime. These sensitive disorders when patients are regaining facial mobility and the mobility of expressions can lead to parasitic or compensatory contractions of the platysma muscles or the incidence of parafunctions (Fig. 15).

The use of transcutaneous electrical nerve stimulation (TENS) or electrotherapy can promote the return of sensations. The presence of material

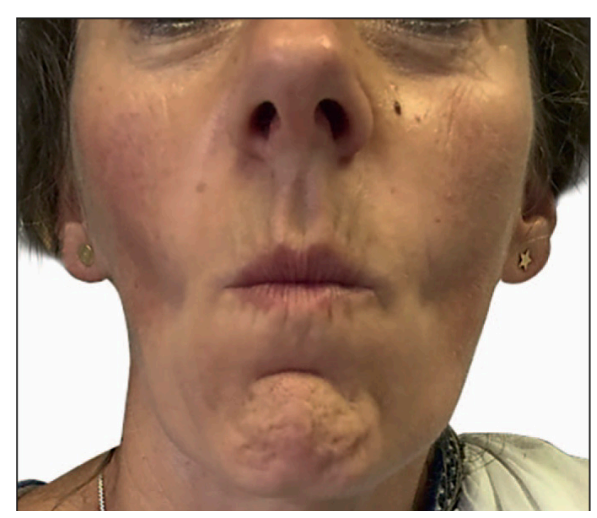

Figure 15

Parafunction appears after hypoesthesia. 
from osteosynthesis indicates the use of polarized electric currents as well as vibratory systems.

Intermaxillary blocking, despite progressive daily withdrawal, provides prolonged dental contact information, whereas the normal duration of contact does not exceed 25-30 minutes maximum for the day. The patient becomes used to this contact while integrating the new occlusion into the body's framework. A type of dental clamping can also be observed. The patient must fight against this parafunction that is similar to centered bruxism, and it generates masticatory muscle tension and causes pain.

The orthodontist now returns to complete the corrections during the 2-3 weeks postoperatively. Ablation of the daily splint, changing the arc or modifying the installed rubber bands can all generate reflex muscle tension on a fully modified area. To improve the patient's comfort as well as his compliance to treatment the orthodontic follow-up schedule must be maintained and muscle relaxation strongly encouraged.

Very often, mandibular excursion recovers with proper kinematic exercises. However, the very important actions must not be forgotten but automatized, such as with mastication or phonation. This gives a natural aspect. The same can be done with a patient who, after lower-limb orthopedic surgery, recovers his mobility and all his force, but continues to limp because the movement was not fully reintegrated.

\section{Ablation of osteosynthesis material}

During their recuperation, patients, especially the more active ones, admit to feeling the effects from the osteotomy in their daily life. For some, once the edema is reabsorbed, touching the plates and titanium screws leads many to request the ablation or removal of the material. According to Mouton ${ }^{5}$, in 2006, they were just isolated cases but at present the request is becoming more and more frequent. In most cases, this request is made 8-14 months after the osteotomies, which means that rehabilitation would usually be complete.

The surgeon can request that the rehabilitator attend the preoperative consultation to ease muscle tension, which will be progressively reinstated after treatment ceases. It can also be planned in postoperatively to reassess the functions, relax residual contractures, or even to develop a precise protocol for autorehabilitation exercises to be done by the patient over time. For example, a patient who presents a strong lingual interposition during treatment and therefore presents a serious risk of relapse. OSAHS symptoms can require upkeep of the tonicity of the velar and retrobasilingual muscles. The final goal being functional automatization.

This post-ablation care structure of the osteosynthesis plates is a lot less systematic and implemented on a case-by-case basis, some patients may not need physical therapy nor myofunctional treatment at this stage. 


\section{THE PSYCHOLOGICAL COMPONENT}

Throughout the treatment, the psychological care of the patient is of the utmost importance. It is, therefore, a long process requiring the psychologist's involvement from start to finish. The role of the rehabilitator is to maintain this motivation throughout. This support allows us to follow the progress of the patient's psychological state.

In the preoperative period, apart from the breathlessness, patients may show signs of anxiety. Specialists must answer all the patient's questions about treatment duration, possible pain, edema, diet, the rubber bands, future physical appearance etc. To allay the worries of patients, each person's consultation must be complementary and must go in one common direction.

In the postoperative period, a frequent sensation of oppression has been described and this can be linked to a number of things: intermaxillary blocking, obstructed airways, compression due to edema, and a lack of intraoral proprioception with the tongue misplaced. The state of fatigue in which the patient finds themselves can also be contributory. Their diet may be less rich and nocturnal discomfort can slow down recovery. Some patients describe an almost immediate aftermath to the surgery.

\section{STUDY}

In daily practice, we are very often confronted with the phenomena of contractures or muscle tension. Described by the patient and manually identified by the rehabilitator, they cause pains and have an impact on
For others, the effects were only felt some days later.

Cutaneous pain relief, following bone displacement, causes a somewhat significant esthetic change. The patient must adapt to this new face and create a new identity. Although the result is always better, it is an external view and so the patient must still be able to recognize themselves in the mirror as well as be recognized by others. At first, the edema, which can be serious, upsets the patient and does not give a positive impression of the new morphology. Vanpoulle ${ }^{37}$ emphasizes that women are more sensitive to this esthetic difference as opposed to men. Furthermore, a significant dysmorphia can bring about a great change that is more easily tolerated than a small displacement because in this case, the patient who was not happy with their old face, adopts the new one more readily.

The rapid reabsorption of edema, the recovery of one's facial expressions, their opinions of themselves when looking in a mirror are all favorable factors. Possible delays in the recovery of sensitivity can have a negative effect. The kinesiologist must make the entire process psychologically effortless by optimizing the positive impact of functional and esthetic results.

the functional progression of the buccal opening as well as the postural balance of the person who is in the process of reconstructing their body image. To try and clarify the importance and structure of these 
muscle phenomena, a study was conducted.

The muscle groups studied are the masticatory muscles: masseter, temporalis, and lateral and medial pterygoids. These powerful mandible elevator muscles often suffer from preoperative tension due to dysmorphia or to lingual dysfunction. After intervention, they have to undergo morphological bone-based changes, intermaxillary blocking, pain, finishing touches and orthodontic treatment. Their care is unanimously acknowledged by all authors after orthognathic surgery. ${ }^{1,5,17,18,34,37}$

Second, the study evaluated the presence of cervical and suboccipital muscle tension as many of the problems brought up by patients are located in this region. The muscles concerned are deep and short, and they extend from the occiput to the first cervical vertebra. There are four per side, rectus capitis anterior, rectus capitis lateralis, obliquus capitis superior, and obliquus capitis inferior, which is in contact with Arnold's nerve. A muscle contracture that is too severe will put tension on the nerve and provoke occipital neuralgias. Sensations of this type have been briefly mentioned in some cases. This suboccipital musculature de- scribed by Barette ${ }^{3}$, is abundant in proprioceptive receptors, has the role of stabilization and the postural positioning of the head within the three planes of space, particularly to maintain the horizontality of sight. They are sensitive to palpation in cases of excessive tension, causing pain in the upper neck. The surface is composed of longer splenius and semispinalis muscles working in a chain ${ }^{24}$. The sternocleidomastoid, occipitalis, and scalene muscles (often excluded when discussing contractures in favor of SCOM) have the job of orienting the head in space. Then we find the muscles linked to the scapula, the upper trapezius muscles, and the circumflex scapular artery. There is also a connection to the face via the occiptofrontalis muscle. The antagonistic muscles situated on the anterior side contribute not only to counterbalancing the ones preceding to ensure postural equilibrium of the head but they are directly linked with the stomatognathic system when it comes to surgery. The surpahyoid and infrahyoid muscular system is the anatomical, postural link between the mandible, the base of the skull, the tongue and the torso, this is achieved by way of relays on the hyoid bones, which is how it earned the name postural "gyroscope"30 (Fig. 16). 


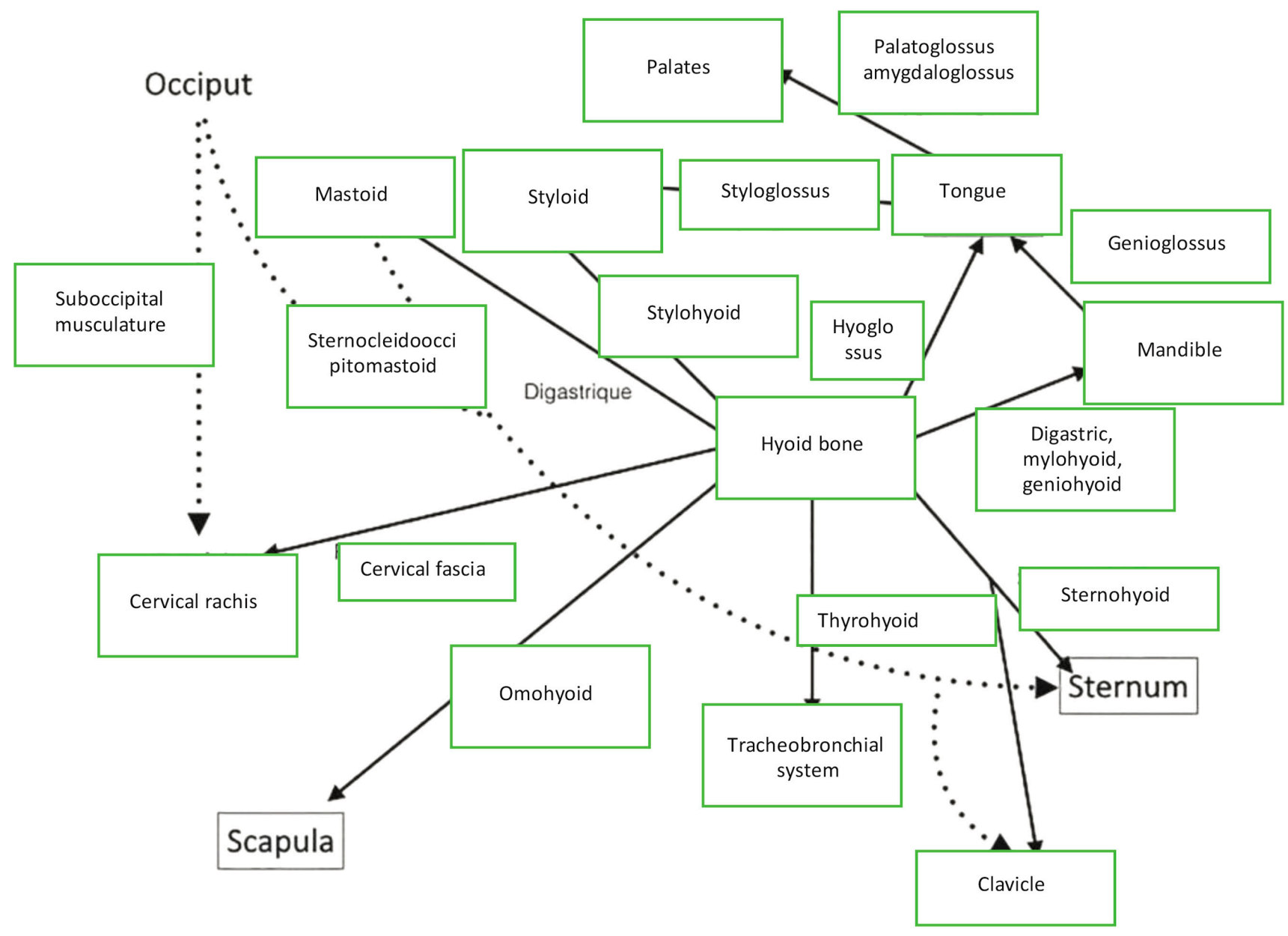

Figure 16

Anatomical connection of the hyoglossal apparatus.

\section{Sample}

The sample studied concerns patients who underwent orthognathic surgery in the form of a maxillary or bimaxillary mandibular osteotomy. Genioplasties and maxillary disjunctions have been excluded, because they do not create sufficient structural modifications. The selected sample for the study contains 35 individuals who were randomly selected among the population in question without discrimination according to sex or age.

\section{Method}

The patients making up the sample were followed from their immediate postoperative rehabilitation to the end of the treatment, over the course of approximately 6 months. The criteria studied were: 
- Preoperative Angle's class (without subdivisions)

- History of muscle tension and neck pains

- The appearance of immediate contractures (during hospitalization or upon the return home)

- Later appearance of contractures

- Tension localization on the masticatory muscles (MAS)

- Tension localization in the cervical and the suboccipital region (CSO).

Data collection was done in part by response to a questionnaire and also by the result of a palpatory analysis done during the consultations. The parameters are subjective but were recorded by the same operator under the same conditions.

\section{Results}

Data collection shows that the masticatory system (MAS) is implicated in $100 \%$ cases (Fig. 17) and most often unilaterally (83\%). Experience shows that there is rarely a change in side during the recovery period, although in some cases it shifts. The CSO region is not systematically prone but all the same it occurs in $74 \%$ cases.

The division of the Angle's classes within the group did not allow us to draw any significant conclusions (Fig. 18). However, that would leave us to suppose that Class III patients $(60 \%)$ are less susceptible to CSO than Class II (77\%).

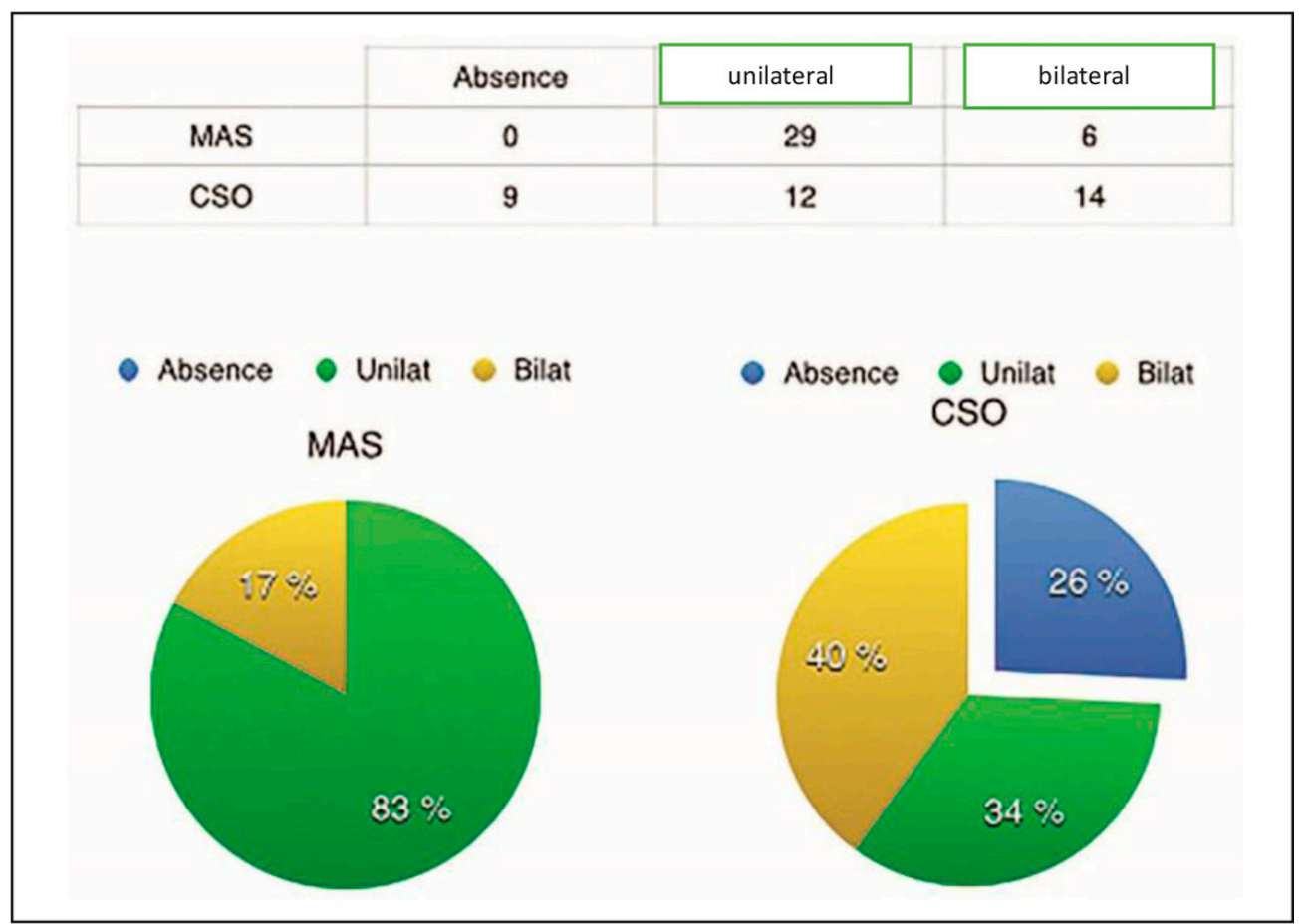

Figure 17

Appearance of muscle tension in the masticatory apparatus and the cervical and suboccipital musculature. 


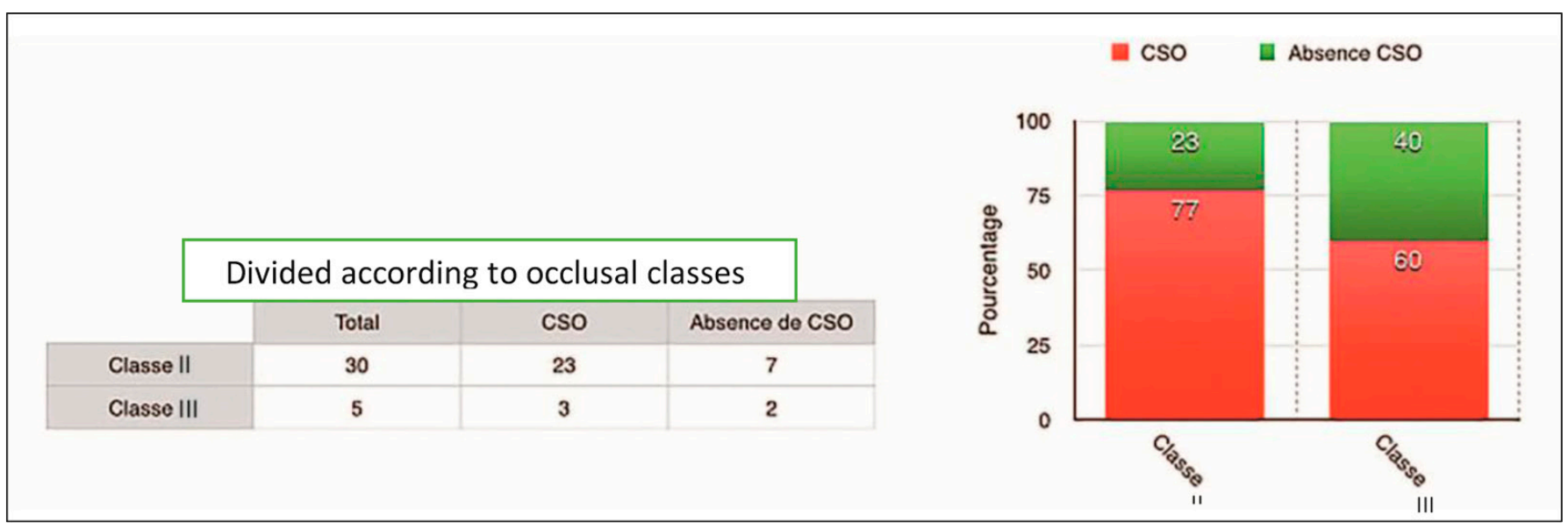

Figure 18

Report CSO muscles tension according to occlusal class.

The lateralization of the CSO correlated to identical MAS movements, in 11 out of 12 cases on the same side, which suggests that there is a link between the two (Fig. 19). It would have been interesting in the cases of bilateral CSO to know if one side was significantly more contracted, to trace a parallel with the corresponding masticatory side.

Of the seven patients having indicated preoperative symptoms, six experienced immediate postoperative pains.

This implies that the fewer contractures patients have because of intervention, the more comfortable their recovery will be.

The final element that stands out is the onset of symptoms within days of the intervention (23 cases), which is almost twice the number shown in immediate postoperative care (12 cases). The preoperative position and the importance of the intervention are not the only factors. This implies a more mechanical factor during the removal of the transfixion and functional stimulation.
CSO Unilatérales

\begin{tabular}{|l|r|}
\hline Homolateral MAS & 11 \\
\hline Contralateral MAS & 1 \\
\hline & 12 \\
\hline
\end{tabular}

Figure 19

Lateralization of CSO muscle tension in relation to MAS.

\section{Discussion}

Although the results appear interesting and by professional practice we could track the correlation quite well but the sample size does not allow us to draw any conclusions especially about the class II to class III proportion. The same can be said for the type of orthognathic surgery because no difference was observed. The preoperative analysis of the morphotype would be interesting to correlate to the lateralization of muscle tension.

Moreover, only the onset of symptoms has been indicated but not there 
is nothing about the resurgence over the course of postoperative treatment. The statements made by the patients refer to events such as the modification of the elastic forces, stopping the splint, changing the arc, or resuming work. The kinesiotherapy follow-up throughout treatment even if spaced out in time has proven to be effective in managing this type of manifestation as well as for ensuring better treatment compliance with shorter delays.

\section{Synthesis}

Although the sample studied was not very large (35 cases), the tendency for muscle tension to appear is very clearly marked in the effects of orthognathic surgery, especially on the masticatory apparatus but also on the suboccipital musculature. The lateralization implies the existence of a correlation between the two. The difference between the preoperative occlusal classes is not at all significant. The direct modification of muscle tension on the anterior muscles by surgery and the onset of posterior cervical contractures suggest a treatment impact beyond that of the masticatory apparatus, via the muscle chains on the rest of the posture. Furthermore, Vaillant ${ }^{35}$ demonstrates the impact of cervical muscles on postural control. The significance of the results of the study confirms the views tied to experience and the need to treat these muscular manifestations for the improved comfort of the patient and also to encourage the postural integration of any facial morphological modification surgery. It seems worthwhile to conduct the study on a larger sample and eventually to include other criteria.

\section{CONCLUSION}

Surgico-orthodontic care specialists, to restore functional and structural equilibrium, will be faced with many anatomical, psychological, and functional disruptions. The kinesiologist, oromaxillofacial rehabilitation specialist, assists in preoperative treatment to ensure that the patient benefits from optimal surgical conditions. The kinesiologist works on the musculoskeletal system and corrects orofacial dyspraxias. In this way, they contribute to making postoperative recovery more comfortable for the patient and optimize recuperation. In the long term, the rehabilitation will automatize the results and limit the risk of relapse. The conclusions of the study conducted on the onset of postoperative muscle tension show that they must not be neglected. This means we need to have a more general vision of the patient and provide them with the best therapeutic solutions. All this can only happen with a multidisciplinary team in which each person plays a key role and the combined efforts help to establish wellcoded protocols.

Conflict of interest: The authors have declared that they do not have any conflict of interest. 


\section{BIBLIOGRAPHY}

1. Alvarado-Fraysse C. Dento-facial orthopedics and kinesthetic therapy: partners in patient management. Orthod Fr 2014;85:275-285.

2. Alvarado-Fraysse C, Breton I, Pommerol P. Dysfonction de I'appareil manducateur et thérapie manuelle. Kinésithér Scient 2012;530:53-56.

3. Barette G, Barillec F, Estampe F, Ghossoub P. Organisation musculaire du rachis cervical. Kinésithér Scient 2013;540:11-16.

4. Bigot $F$, Hadjadj M. Les fondamentaux en rééducation OMF Bilan et technique. Kinéactu 2011;1249:56-60.

5. Borel-Maissonny S. La déglutition dysfonctionnelle. Paris: Rééducation orthophonique, 2006:226.

6. Clauzade M. Orthoposturodontie. Actualités o dontostomatologiques 2007; 240:387-405.

7. Duez D. Le traitement des œdèmes après chirurgie cervico-faciale et maxillo-faciale. Kinésithér Scient 2003;432:23-26.

8. Engelke W, Jung K, Knösel M. Intra-oral compartment pressures: a biofunctional model and experimental measurements under different conditions of posture. Clin Oral Investig 2011;15:165-176.

9. Faulin EF, Guedes CG, Feltrin PP, Joffiley CM. Association between temporomandibular disorders and abnormal head postures. Braz Oral Res 2015;29:1-6.

10. Fournier MY, Chauvois A, Girardin M. Rééducation des fonctions dans la thérapeutique orthodontique. Paris: SID éd., 1993.

11. Fournier M. Le rôle du rééducateur. L'information dentaire 2009;25:1402-1404.

12. Gallerano G, Ruoppolo G, Silvestri A. Myofunctional and speech rehabilitation after orthodontic-surgical treatment of dento-maxillofacial dysgnathia. Prog Orthod 2012;13:57-68.

13. Girard M, Leroux C. Gestion des muscles et des fonctions par le kinésithérapeute dans les traitements orthodontiques et ortho-chirurgicaux. Rééducation oro-myofonctionnelle. Orthod Fr 2015;86:95-111.

14. Guimaraes KC, Drager LC, Genta PR, Marcondes BF, Lorenzi-Filho G. Effects of oropharyngeal exercises on patients with moderate obstructive sleep apnea syndrome. J Respir Crit Care Med 2009;179:962-966.

15. Houb-Dine A, Bahije L, Zaoui F. La rééducation linguale et la stabilité occlusale chez le jeune adulte. Rev Mens Suisse Odontostomatol 2012;122:529-534.

16. Jouvin B. Kinésithérapie mandibulo-faciale. Paris: Maloine Ed., 1985.

17. Jung HD, Jung YS, Park JH, Park HS. Recovery $p$ attern of mandibular movement by active physical therapy after bilateral transoral vertical ramus osteotomy. J Oral Maxillofac Surg 2012;70(7):e431-437.

18. Ko EW, Teng TT, Huang CS, Chen YR. The effect of early physiotherapy on the recovery of mandibular function after orthognathic surgery for class III correction. Part II: electromyographic activity of masticatory muscles. J Craniomaxillofac Surg 2015;43: 138-143.

19. LaBanc JP, Epker BN. Changes of the hyoid bone and tongue following advancement of the mandible. Oral Surg Oral Med Oral Pathol 1984;57:351-356.

20. Makaremi M, Zink K, de Brondeau F. Apport des contraintes masticatrices fortes dans la stabilisation de l'expansion maxillaire. Rev Orthop Dento Faciale 2015;49:1-10.

21. Manas-Gomez F, Lozano V, Guatterie M. Drainage lymphatique cervical. Kinésithér Scient 1995;343:23-27. 
22. Maniere-Ezvan A, Tamine F. Croissance et rééducation fonctionnelle oro-faciale: le rôle de I'omnipraticien. Réalités cliniques 2005;16:5-20.

23. Marchesan IQ. Lingual frenulum: quantitative evaluation proposal. Int J Orofacial Myology 2005;31:39-48.

24. Matheron E, Weber B. Approche de la relation ATM et chaînes musculaires. Kinésithér Scient 2006;472:33-38.

25. Moeller JL, Coceani Paskay L, Gelb ML. Myofunctional therapy. A novel treatment of pediatric sleep-disordered breathing. Sleep Med Clin 2014;9:235-243.

26. Phillips C, Kim SH, Essick G, Tucker M, Turvey TA. Sensory retraining after orthognathic surgery: effect on patient report of altered sensations. J Orthod Dentofacial Orthop 2009;136(6): 788-794.

27. Quesnot $A$, Chanussot JC, Corbel I. La cryothérapie en rééducation: revue de littérature. Kinésithér Scient 2001;416:21-29.

28. Raberin M. Incidences cliniques de la zone orolabiale. EMC (Elsevier Masson SAS, Paris), Odontologie/ Orthopédie dentofaciale, 23-474-B-10, 2007.

29. Rosenthal G. Rééducation des obstrués du nez et rhino-pharynx. Paris: G. Doin et Cie, 1933.

30. Scoppa F. Glosso-postural syndrome. Annali di Stomatologia 2005;LIV(1):27-34.

31. Susanibar F. Phonoaudiological assessment of respiration. Italy: LCF Edizizioni, 2014.

32. Takahashi S, Kuribayashi G, Ono T, Ishiwata Y, Kuroda T. Modulation of masticatory muscle activity by tongue position. Angle Orthod 2005;75(1):35-39.

33. Teng TT, Ko EW, Huang CS, Chen YR. The Effect of early physiotherapy on the recovery of mandibular function after orthognathic surgery for Class III correction: part l--jawmotion analysis. J Craniomaxillofac Surg 2015;43:131-137.

34. Trawitzki LV, Dantas RO, Mello-Filho FV, Marques W Jr. Masticatory muscle function three years after surgical correction of class III dentofacial deformity. Int J Oral Maxillofac Surg 2010;39:853-856.

35. Vaillant J, Pinsault N, Vuillerme N, Gros G, Rousset R. Implication du rachis cervical dans le contrôle de la posture: des évidences expérimentales aux conséquences pratiques. Kinésithér Scient 2006;467:29-39.

36. Valdés $C$, Astaburuaga F, Falace D, Ramirez V, Manns A. Effect of tongue position on masseter and temporalis electromyographic activity during swallowing and maximal voluntary clenching: a cross-sectional study. J Oral Rehabil 2014;41(12):881-889.

37. Vanpoulle F. Intérêt de la prise en charge en kinésithérapie oro-maxillo-faciale des chirurgies orthognathiques. Kinésithér Scient 2005;460:23-35.

38. Vanpoulle F. La posture linguale a-t-elle une influence sur la performance sportive? Kinésithér Scient 2013;544:17-26. 